\title{
Characteristics of hip fractures due to minimal trauma in older people of a health area in Southwest Spain
}

\begin{abstract}
Osteoporotic hip fracture is one of the pathologies of the elderly with greatest morbidity and mortality, dependency, and health costs. Therefore, the objective of this study was to identify some of the determinants of minimal trauma hip fracture in patients of 65 or older. A descriptive, prospective, transversal study was conducted based on a structured survey addressed to the 233 patients admitted to the Virgen del Puerto Hospital for hip fracture in two years. The patients belonged to a health care zone of 70 rural municipalities (Plasencia, Cáceres, Spain) in the years 2007 and 2008. The largest number of fractures occurred in women of $>80$ years $(\mathrm{p}=0.005)$ when they were turning around or standing up or sitting down, did not use elements of support for walking $(\mathrm{p}<0.005)$, had suffered some previous fracture $(\mathrm{p}=0.0005)$, led a sedentary lifestyle, had calcium-poor diets, and lacked health information on this pathology and its prevention. These results allowed identifying the characteristics of hip fractures as women older than 80 , with a sedentary lifestyle, a calcium intake below recommended levels and who resist using canes or crutches as aids to walking.
\end{abstract}

Keywords: hip fractures, osteoporosis, aged, community health nursing, Nursing assessment prevention
Volume I Issue 5 - 2017

\author{
Gómez González Ma Ángeles,' Pérez \\ Pico Ana María,' Martínez Nova Alfonso,' \\ Regueira Daza Jesús, ${ }^{2}$ Raquel Mayordomo \\ Acevedo 3 \\ 'Departamento de Enfermería, Universidad de Extremadura, \\ Spain \\ 2Departamento de Ciencias Biomédicas de la Universidad de \\ Extremadura, Spain \\ ${ }^{3}$ Departamento de Anatomía y Biología Celular de la \\ Universidad de Extremadura, Spain
}

Correspondence: Raquel Mayordomo Acevedo, Lecturer at the Department of Anatomy, University of Extremadura, Spain, Tel +34636526498, Fax +34927425209, Email rmayordo@unex.es

Received: March 31, 2017| Published: July 25, 2017

\section{Introduction}

Osteoporotic hip fracture is a serious public health problem both because of the advanced age of the typical patient and the major fraction of health care resources that it consumes. The former is the reason for its high mortality ${ }^{1,2}$ and that it results in major functional losses. ${ }^{3,4}$ The latter means that it constitutes a major economic burden on the health care systems of all developed countries, with there still existing no effective protocols for its detection and prevention. ${ }^{5,6}$

Approximately $85 \%$ of these fractures occur in patients older than $65^{7}$ so that its global incidence will continue to rise together with the aging of the world's population. In its origin, one distinguishes two clear aspects. One is the predisposition represented by the fragility of de-mineralized bone ${ }^{8}$ and the other is the trigger represented by the trauma or impact the bone was subjected to that caused it to break. The former aspect includes, besides sex (females) and age ${ }^{9}$ multiple risk factors. While some of these factors are genetic, others are lifestyle related- exercise, calcium intake, toxic habits, etc... ${ }^{10}$ Which for the same age and sex, determine different degrees of osteoporosis. Regarding the latter aspect, falls have been identified as the main cause of these fractures. ${ }^{11}$ The objective of the present work was to determine how much this pathology is influenced by some of these risk factors, thus obtaining a profile of the hip fractured elderly in our health care zone based on their habits and lifestyle. A further objective was to determine whether the underlying cause was really a fall, or whether this fall itself occurred as a result of a previous fracture of the bone. This should thus contribute to our profession's developing prevention protocols aimed at reversing or blocking those risk factors which are modifiable ${ }^{12}$ given that intervention strategies come to a great part under the remit of nursing. ${ }^{13}$

\section{Materials and methods}

\section{Study design and population}

A descriptive, prospective, transversal study was conducted based on a structured survey ${ }^{14}$ addressed to the 233 patients admitted to the Virgen del Puerto hospital for hip fracture in two years. Their mean age was $82.89 \pm 7.21$ years (range $65-102$ years), a female-to-male ratio of 3.48:1 (77.68\% women, $22.31 \%$ men). In 2007 there were 112 fractures and in 2008 there were 121, which means an annual increase of $3.87 \%$.

\section{Inclusion criteria and procedure}

Patients of 65 and older with a diagnosis of minimal trauma hip fracture belonging to the Plasencia health care zone in south-west Spain, at latitude 40 north. The medical records were reviewed of all patients admitted to the traumatology unit to assess the clinical aspects of the fractures. The same investigator interviewed them based on a structured questionnaire comprising sociodemographic, diet, mobility, habits, and lifestyle items in 233 patients and/or families. Dairy product intake was evaluated in terms of a continuous variable calculated mainly based on the daily milk consumption and the weekly consumption of yoghurt and portions of cheese..$^{15}$

\section{Permissions}

The necessary permissions were obtained from the hospital's administration and Bioethics Committee $(\mathrm{Id}=14 / 07 / 2009)$. All the patients were explained the purpose of the study, and acceptance was required in the form of their informed consent. 


\section{Statistical analysis}

All values are expressed as mean \pm standard deviation (SD). Normality of the distribution of the data was confirmed by calculating skewness and kurtosis before applying the standard test. For a confidence interval of $95 \%$, the level set for statistical significance was $p<0.05$. The data were analyzed by either an analysis of variance (ANOVA) or Student's t test, as appropriate. Associations between the variables were examined using simple and multiple regression analyses and Pearson's correlation coefficient, and, in the case of percentages, a chi-squared test. All statistical analyses were done using the Stat View 5.0.1 software package (SAS Institute Inc., Cary, NC, USA).

\section{Results}

The mean age was $82.89 \pm 7.21$ years (range $65-102$ years) and there were no significant differences in the presentation of fractures for both sexes, being $83.28 \pm 7.07$ years for women and $81.53 \pm 7.57$ years for men. Of the total fractures, $77.68 \%$ are women and a $22.31 \%$ in men, which means a women/men ratio of 3.48: 1. Figure 1 shows how fractures increase with age in both sexes but especially in women. Fractures, occurred in women and more in the older age group, i.e., those aged 81 years or older (153 patients). The fractures occurred during the day in $81.97 \%$ of the cases, and in home in $61.80 \%$, with $13.3 \%$ occurring in nursing reception or similar geriatric institutions Table 1 Falls constituted the primary cause (57\% of cases), followed by spontaneous fracture $(38.19 \%$, with a greater proportion in the women, i.e., $39.77 \%$ of the women vs $32.69 \%$ of the men). Those who fell identified the different immediate causes of their fall as specified in Figure 2. One observes in the figure that 83 patients reported making a simple turn as being the cause, or changing position in the sense of simply standing up or sitting down. Spontaneous fracture was more frequent in patients with limited mobility $(53.03 \%)$ than in those with normal mobility $(\mathrm{p}<0.0001)$.

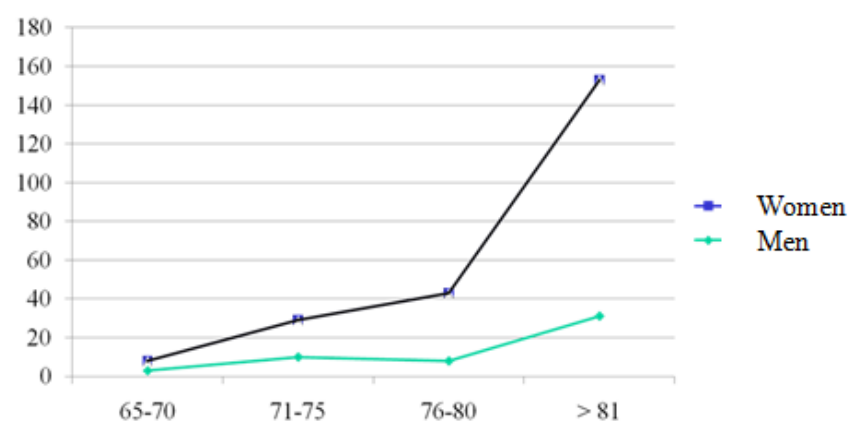

Figure I Number of fractures per age range. This figure shows that in both cases, but especially in the group of women, the incidence of falls increases gradually with age.

Table I Incidence of fractures by age range. The numbers of fractures are higher in women than in men and also correlates with the age

\begin{tabular}{llllll}
\hline Sex & $\mathbf{6 5 - 7 0}$ & $\mathbf{7 1 - 7 5}$ & $\mathbf{7 6 - 8 0}$ & $\mathbf{> 8 1}$ & Total \\
\hline Men & 3 & 10 & 8 & 31 & 52 \\
Woman & 5 & 19 & 35 & 122 & 181 \\
Total & 8 & 29 & 43 & 153 & 233 \\
\hline
\end{tabular}

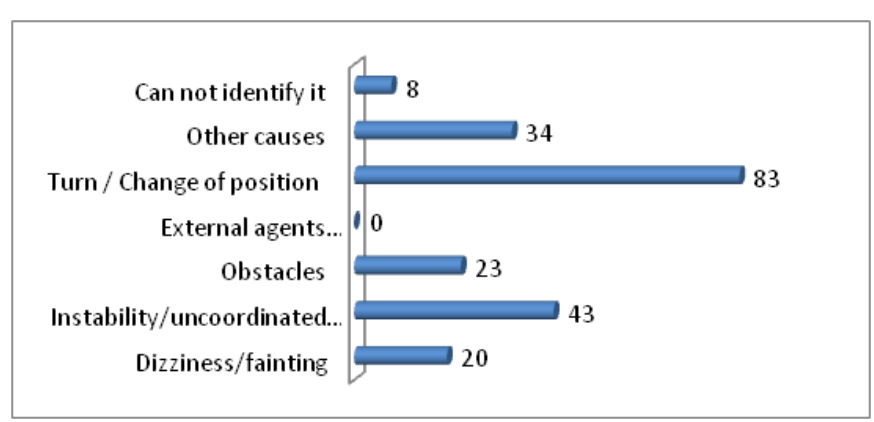

Figure 2 Cause of fall or fracture. In the bars the data show that turn or change of position is the most prevalent cause of fracture in the study population. Also instability is the cause of a high number of fractures.Very few fractures are cause by external factors as footwear.There were significant differences by sex in where the fracture occurred. More happened in the street in men than in women $(\mathrm{p}=0.005)$. This could be explained by the men's greater practice of physical activity $(p=0.0355)$.

With respect to the relationship of the use of external support in walking with the falls, there were more falls when no support was being used $(\mathrm{p}<0.0001)$ especially for women (Table 2$)$.

Table 2 Use of supports or external aids. For man and woman cane or crutch are the most usual supports for held on walking (more for men than for woman). Notice that wheelchair is preferred by woman better than for man if they need it, but in general are woman who avoid taking any support at all. NK/NR, no knowledge/no response. N, number. \%, percentage

\begin{tabular}{llll}
\hline $\begin{array}{l}\text { Use of supports or } \\
\text { external aids }\end{array}$ & $\begin{array}{l}\text { Total \% } \\
\mathbf{N}=\mathbf{2 3 3}\end{array}$ & $\begin{array}{l}\text { Man } \\
\text { \%N=52 }\end{array}$ & $\begin{array}{l}\text { Woman } \\
\% \mathbf{N}=18 \text { I }\end{array}$ \\
\hline Cane / Crutch & 52.36 & 69.23 & $47.5 \mathrm{I}$ \\
Walker / Wheelchair & $\mathrm{II} .15$ & 5.76 & 12.7 \\
None & 33.04 & 25 & 35.35 \\
NK/NR & 3.43 & 0 & 4.42 \\
\hline
\end{tabular}

No physical exercise was performed by $57.08 \%$ of the subjects. Only the $36,4 \%$ of participants did referred to walking for half an hour a day, while only $3 \%$ performed some other activity such as mild exercise or cycling. A greater proportion of women than men did no exercise $(60.22 \%$ vs $46.15 \%)$. A greater proportion of men walked daily had a higher rate of fracture from falls than men who do no physical exercise $(26,26 \%$ vs $8,75 \%$, $\mathrm{p}=0.0001)$.

The mean calcium intake was $715.27 \pm 312.56 \mathrm{mg}$ /day (range 0 $1635 \mathrm{mg}$ /day). There were no significant differences between men and women, and there was a decrease in this parameter with increasing age (Figure 3).

Almost all the men reported alcohol and smoking habits. Although most of them no longer smoked at the time of the fracture and drank moderately ( $<3$ glasses/day), $61 \%$ had previously been heavy smokers. With respect to alcohol habits, $18,02 \%$ of the patients drink alcohol. Most of those who drink were men (36 from 52 men with fractures) and 18 of then stated that they were ex-heavy-drinkers $(34,6 \%)$, and four cases were detected of a history of alcoholism.

A previous fracture had been suffered by $30.47 \%$ of the patients, with the proportion being significantly higher in women $(35.91 \%$ vs $11.53 \%, \mathrm{p}=0.0005)$. The commonest site of those previous fractures 
was the hip, followed by the upper limb. Early menopause was detected in $18.47 \%$ of women, and was correlated with the fracture occurring at a younger age $(p=0.0005)$. Regarding the subjective state of health before the fracture, most of the patients positioned themselves in the good categories rather than the bad (Table 3).

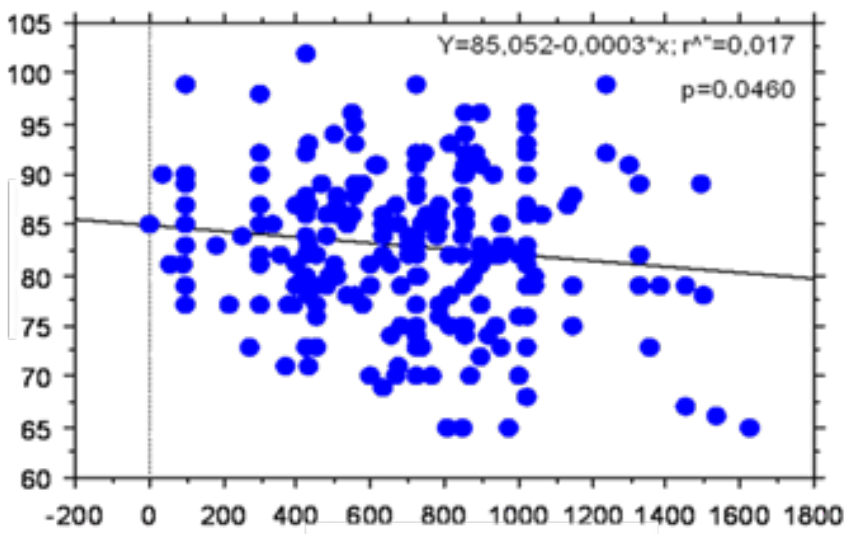

Figure 3 Linear regression of calcium intake $(\mathrm{mg} /$ day) and age. The figure shows how calcium intake decreases with age and there is a not difference between men and women.

Table 3 Subjective state of health. The table collects the different categories of state of health according to INE scale (2008)

\begin{tabular}{llll}
\hline $\begin{array}{l}\text { Subjective } \\
\text { state of health }\end{array}$ & $\begin{array}{l}\text { Total \% } \\
\mathbf{N}=\mathbf{2 3 3}\end{array}$ & $\begin{array}{l}\text { Man } \\
\text { \%N=52 }\end{array}$ & $\begin{array}{l}\text { Woman } \\
\% \mathbf{N}=18 \text { I }\end{array}$ \\
\hline Very good & 11.63 & 11.53 & 11.66 \\
Good & 31.46 & 26.92 & 32.77 \\
Regular & 30.17 & 32.69 & 29.44 \\
Poor & 16.81 & 23.07 & 15 \\
Very poor & 1.29 & 0 & 1.66 \\
NK/NR & 8.62 & 5.76 & 9.44
\end{tabular}

NK/NR no knowledge/no response; $\mathrm{N}$ number. \%, percentage.

With respect to health care information concerning the risk of fractures, only $6 \%$ reported having received some advice from their general practitioner (GP), while $74 \%$ stated that they had no knowledge about it. Only $24.30 \%$ of the women and $9.6 \%$ of the men knew something about it $(\mathrm{p}=0.0080)$.

\section{Discussion}

In Spain the evolution of positive self-assessment of health has risen slightly; From $68.8 \%$ of the population considered to be in good or very good health in $1993,69.8 \%$ in 2001 , to $70.0 \%$ in 2006 . In $2006,75 \%$ of the men said they had good or very good health, compared to $65 \%$ of women. The scale used by the INE in the report the elderly in Spain collects the categories of very good, good, regular, bad and very bad (page 97, consultation in June 2008).

In our study, patients who consider their health status within the first three categories, including that of regular, amounts to $73.26 \%$, so we can conclude that this self-perception is more good than bad, despite the old age from the patients. Besides, the prevalence of fractures in women is fully in line with many previous studies, which report that women account for as many as $72 \%$ of all these fractures. This is explained by their both attaining a lower peak bone mass, and undergoing rapid bone mass loss with menopause or estrogen deprivation. ${ }^{16}$ A Simple explanation would be that women have a longer life expectancy than men and therefore has more probability of falls and have a fracture because they have all the named factors associated (osteoporosis, menopause, loss of mass bone....) and in a longer period of time.

Regarding age, our results also confirm that the incidence increases gradually from 65 to 95 years of age ${ }^{17}$ and the mean age above 80 years ( $82.89 \pm 7.21$ years) would be consistent with the increasing longevity in our society leading to older patients suffering fractures because of osteoporosis (Figure 1).

Falls were identified as the primary cause of fracture, in line with other studies. ${ }^{18}$ Fall were referred and was main part $(35.62 \%)$ a simple turn or change in position, whereas other workers report the commonest causes as being due to external agents, such as tripping or slipping on something. ${ }^{19}$ This, together with the $38.19 \%$ of patients who reported that the fracture occurred spontaneously, is evidence of the fundamental part played by osteoporosis in these fractures. There is further support for this in that the fractures occurred primarily in women and in persons with limited mobility, the former reflecting their greater propensity to bone mass loss and the latter because longterm immobility also causes a decrease in bone mass. ${ }^{20}$ That the men had a greater proportion of falls as the cause of their fracture could be explained by their greater physical activity. This in turn is related to the housewife function that women traditionally exercise, and to other social connotations, ${ }^{21}$ although one also finds in the literature results that point in the opposite direction, with a higher incidence of falls among women. ${ }^{22}$ There were more falls in the subjects, which not used external supports for ambulation. While this is coherent with the study of Masoni et al. who found mechanical assistance in ambulation to be a protective factor against hip fractures ${ }^{21}$ a greater number of studies have reached conclusions to the contrary, with such assistance being positively correlated with the risk of falling. ${ }^{23}$

A history of previous fractures is one of the most important risk factors for all osteoporotic fractures. ${ }^{24}$ In the present study, a major proportion of the patients $(30.47 \%)$ had suffered a previous lowimpact fracture after the age of 50. This percentage was much higher in women than in men $(p=0.0005)$, and hip fractures were the most frequently repeated. This is consistent with the fact that a history of a previous fragility fracture is an important risk factor for this pathology. ${ }^{25}$

In this work is shown that the group of women patients who had not been treated with hormone replacement therapy or antiresorption drugs suffered the fracture at an earlier age than the rest of the women $(\mathrm{p}=0.0005)$. These results and other studies ${ }^{26}$ suggest, that early menopause could be a risk factor for osteoporosis based on fractures due to deprivation of hormone replacement therapy. Besides, the National Osteoporosis Foundation guidelines for the assessment, diagnosis, prevention and treatment of osteoporosis identify hormone replacement therapy as a first-line therapy from year 1998. ${ }^{27,28}$

The physical inactivity, more evident in the women, also reinforces the conclusion that moderate levels of activity are associated with a substantially lower risk of fracture. Indeed, exercise has a beneficial effect not only on both bone mineral density, but also on muscle strength, balance, and coordination. The Study of Karlsson ${ }^{20}$ Finds 
that this significantly reduces falls, although others ${ }^{29}$ fail to find any such association.

Calcium intake in the patients' diet was well below the recommendation $1500 \mathrm{mg} /$ day for the elderly. This has also been noted in other studies ${ }^{30}$ with a decline in consumption as age increases. This reinforces the fact that calcium deficiency is a risk factor for osteoporosis, and is significantly associated with hip fracture. ${ }^{31}$

With respect to toxic habits, $34,6 \%$ of men were heavy drinkers (4 cases had a medical history of alcoholism). These data suggest together with other studies ${ }^{32,33}$ that chronic alcohol consumption could be associated directly with the risk of hip fractures.

A major percentage of the patients felt healthy prior to the fracture; consistent with an IMSERSO report, $201,{ }^{34}$ such self-appraisal is a simple but effective indicator of people's overall health status. The extra knowledge it provides on the target population's profile reinforces the epithet applied to osteoporosis of being a "silent epidemic". The knowledge and information gained in this study should help contribute to satisfying the recognized need for appropriate protocols centered on the prevention of the pathology rather than solely on its cure. ${ }^{35,36}$

\section{Conclusion}

The present results allowed us to identify the characteristics hip fractures, as a woman older than 80 , with a sedentary lifestyle, a calcium intake below recommended levels, and who resist using canes or crutches as aids to walking. Indeed, they have previously suffered a hip fracture, and they had early menopause. The man characteristics are; consumer of alcohol or tobacco immoderately, has a calciumpoor diet, and walk daily. Both groups lack knowledge and health information about this pathology and its consequences. It is necessary therefore to improve education and raise awareness about the problem, if not in the entire elderly population, as the characteristics identified.

Despite the high personal, social, and economic cost of hip fracture in the elderly, our health care system is still limited to its care and surgical treatment. It would be far more cost effective to develop prevention protocols in which the nursing profession will have to play an essential role, identifying the factors responsible for the pathology that are reversible or modifiable, designing diets with adequate calcium intake, physical exercise, avoiding toxic habits, the use of external support for walking, etc.

\section{Acknowledgements}

Authors want to thanks to Dr Juan Diego Pedrera Zamorano and Dr Jesus Santos Velasco for their support and orientation of the work.

\section{Conflict of interest}

The authors declare that they have no competing interests. None of the authors has any commercial or financial involvements in connection with this study that represent or appear to represent any conflicts of interest.

\section{References}

1. Pande I, Scott DL, O Neill TW, et al. Quality of life, morbidity, and mortality after low trauma hip fracture in men. Ann Rheum Dis. 2006;65(1):87-92.

2. Hershkovitz A, Polatov I, Beloosesky Y, et al. Factors affecting mortality of frail hip-fractured elderly patients. Arch Gerontol Geriatr. 2010;51(2):113-116.
3. Tsuboi M, Hasegawa Y, Suzuki S, et al. Mortality and mobility after hip fracture in Japan: a ten-year follow-up. $J$ Bone Joint Surg Br. 2007;89(4):461-466.

4. Huang TT, Acton GJ. Ways to maintain independence among Taiwanese elderly adults with hip fractures: a qualitative study. Geriatr Nurs. 2009;30(1):28-35.

5. Manzarbeitia J. Análisis: Costes de las fracturas de cadera Revista Española de Economía de la Salud. 2003;4(4):316-217.

6. Branco JC, Felicíssimo P, Monteiro J. Epidemiology of hip fractures and its social and economic impact. A revision of severe osteoporosis current standard of care. Acta Reumatol Port. 2009;34(3):475-485.

7. Parker M, Johansen A. Hip fracture. BMJ. 2006;333(7557):27-30.

8. Bouxsein ML. Bone quality: where do we go from here? Osteoporos Int. 2003;14(5):118-127.

9. Zamorano PJD, Mohedano BJL, García LJM, et al. Incidence and prevalence of hip fractures in the province of Caceres and their evolutionary trend. Rev Clin Esp. 2004;204(9):448-451.

10. LaFleur J, McAdam MC, Kirkness C, et al. Clinical risk factors for fracture in postmenopausal osteoporotic women: a review of the recent literature. Ann Pharmacother. 2008;42(3):375-386.

11. Formiga F, Pujol R. Mortality associated with femur fractures: analyzing the causes of an old problem. Medicina Clínica. 2005;124(2):55-56.

12. Vestergaard K, Andersen G, Gottrup H, et al. Lamotrigine for central post stroke pain: a randomized controlled trial. Neurology. 2001;56(2):184-190.

13. Lappe JM. Prevention of hip fractures: a nursing imperative. Orthopaedic Nursing. 1998;17(3):15-24.

14. Camarero Rioja LA, Del Val Cid C. Técnicas avanzadas de investigación social. UNED, Madrid, Spain; 2000.

15. Moreiras O, Carvajal A, Cabrera L, et al. Tablas de composición de alimentos Madrid. Ediciones Pirámide, Spain; 2005.

16. Wainwright SA, Marshall LM, Ensrud KE, et al. Hip fracture in women without osteoporosis. J Clin Endocrinol Metab. 2005;90(5):2787-2793.

17. Alvarez NML, Jiménez AB, Rodríguez P, et al. Epidemiology of hip fractures in the elderly in Spain. An Med Interna. 2002;19(8):389-95.

18. Ferrández Portal L. Fracturas en el anciano. Madrid, Sociedad Española de Cirugía, Ortopedia y Traumatología. (SECOC). Aventis Pharma; 2001.

19. De Cabo Rodriguez JA. Epidemiología, modificaciones morfológicas y socio-familiares de las fracturas de la extremidad proximal de Fémur en la población mayor de 69 años en Salamanca durante 1995-1996. Medicina [PhD Thesis]. 2001;228-230.

20. Karlsson MK, Nordqvist A, Karlsson C. Physical activity, muscle function, falls and fractures. Food Nutr Res. 2008;52.

21. Masoni A, Morosazo M, Tomat MF, et al. Association between hip fractures and risk factors for osteoporosis. Multivariate analysis. Medicina (B Aires). 2007;67(5):423-428.

22. Hagino $\mathrm{H}$. Muscle and bone health as a risk factor of fall among the elderly. Epidemiology of falls and fractures. Clinical Calciu. 2008;18(6):747-753.

23. Cooper C, Barker DJ, Wickham C. Physical activity, muscle strength, and calcium intake in fracture of the proximal femur in Britain. $B M J$. 1998;297(6661):1443-1446.

24. Kanis JA, Johnell O, De Laet C, et al. A meta-analysis of previous fracture and subsequent fracture risk. Bone. 2004;35(2):375-82. 
25. Nguyen ND, Pongchaiyakul C, Center JR, et al. Identification of highrisk individuals for hip fracture: a 14-year prospective study. $J$ Bone Miner Res. 2005;20(11):1921-1928.

26. Ghazi M, Roux C. Hormonal deprivation therapy-induced osteoporosis in postmenopausal women with breast cancer. Best Pract Res Clin Rheumatol. 2009;23(6):805-811.

27. Hammond CB, Rackley CE, Fiorica J, et al. Consequences of estrogen deprivation and the rationale for hormone replacement therapy (Review). AJ Manag care. 2000;6(14S):746-760.

28. National Osteoporosis Foundation. Physician s Guide to prevention and treatment of osteoporosis. Washington DC, USA; 1998.

29. Gregson CL, Carson C, Amuzu A, et al. The association between graded physical activity in postmenopausal British women, and the prevalence and incidence of hip and wrist fractures. Age and Ageing. 2011;39(5):565-574.

30. Arana AE, Gutierrez II, Ecenarro MA, et al. Prevalence of certain osteoporosis-determining habits among post menopausal women in the Basque Country, Spain, in 2003. Revista Española de Salud Public. 2007;81(6):647-656.
31. Nurmi LI, Lüthje P, Kaukonen JP, et al. Post-fracture prescribed calcium and vitamin D supplements alone or, in females, with concomitant antiosteoporotic drugs is associated with lower mortality in elderly hip fracture patients: a prospective analysis. Drugs Aging. 2009;26(5):409-421.

32. Mukamal KJ, Robbins JA, Cauley JA, et al. Alcohol consumption, bone density, and hip fracture among older adults: the cardiovascular health study. Osteoporos Int. 2007;18(5):593-602.

33. Holmberg AH, Johnell O, Nilsson PM, et al. Risk factors for hip fractures in a middle-aged population: a study of 33,000 men and women. Osteoporos Int. 2005;16(12):2185-2194.

34. IMSERSO (Instituto de Migración y Servicios sociales). Las personas mayores en España. Spain; 2009.

35. Boixadera I, Vendrell M, Antón I, et al. Valoración del conocimiento de los ancianos sobre la osteoporosis. Justificación de la necesidad de un programa de prevención de fracturas. Enfermería Global. 2004;3(2):1-8.

36. Juby AG, Wenceslau DGCM. Evaluation of osteoporosis treatment in seniors after hip fracture. Osteoporos Int. 2002;13(3):205-210. 